\title{
Herd Immunity Effects in Cost-Effectiveness Analyses among Low- and Middle-Income Countries
}

\author{
Siyu Ma ${ }^{1} \cdot$ Tara A. Lavelle $e^{1,2} \cdot$ Daniel A. Ollendorf ${ }^{1,2} \cdot$ Pei-Jung Lin ${ }^{1,2}$
}

Accepted: 5 December 2021 / Published online: 10 January 2022

(c) The Author(s) 2021

\begin{abstract}
Background Herd immunity (HI) is a key benefit of vaccination programs, but the effects are not routinely included in costeffectiveness analyses (CEAs).

Objective This study investigated how the inclusion of HI in CEAs may influence the reported value of immunizations in low- and middle-income countries (LMICs) and illustrated the implications for COVID-19 immunization.

Methods We reviewed immunization CEAs published from 2000 to 2018 focusing on LMICs using data from the Tufts Medical Center CEA Registries. We investigated the proportion of studies that included HI, the methods used, and the incremental cost-effectiveness ratios (ICERs) reported. When possible, we evaluated how ICERs would change with and without HI.

Results Among the 243 immunization CEAs meeting inclusion criteria, 44 studies (18\%) included HI. Of those studies, $11(25 \%)$ used dynamic transmission models, whereas the remainder used static models. Sixteen studies allowed for ICER calculations with and without $\mathrm{HI}(n=48$ ratios). The inclusion of $\mathrm{HI}$ always resulted in more favorable ratios. In 20 cases (42\%), adding HI decreased the ICERs enough to cross at least one or more common cost-effectiveness benchmarks for LMICs. Among pneumococcal vaccination studies, including HI in the analyses decreased seven of 24 ICERs enough to cross at least one cost-effectiveness benchmark.

Conclusion The full value of immunization may be underestimated without considering a scenario in which HI is achieved. Given the evidence in pneumococcal CEAs, COVID-19 vaccine value assessments should aim to show ICERs with and without $\mathrm{HI}$ to inform decision-making in LMICs.
\end{abstract}

\section{Key Points for Decision Makers}

Despite recommendations from the WHO to include herd immunity in vaccine economic evaluations in LMICs, less than one-fifth of published vaccine CEAs do so.

Including herd immunity effects in vaccine CEAs leads to more favorable CEA ratios.

In $42 \%$ of cases, including herd immunity in CEAs reduces ICERs enough to cross commonly cited LMIC cost-effectiveness benchmarks.

Vaccine value assessments should investigate cost-effectiveness with and without $\mathrm{HI}$ to inform decision-making in LMICs.

\section{Siyu Ma}

masy0318@hotmail.com

1 The Center for the Evaluation of Value and Risk in Health (CEVR), The Institute for Clinical Research and Health Policy Studies, Tufts Medical Center, 800 Washington Street, Boston, MA 02111, USA

\section{Background}

Mass vaccination campaigns can greatly reduce the spread, morbidity, and mortality of many infectious diseases such as influenza [1-4], rotavirus [5, 6], and pneumococcal disease $[4,7]$. Vaccines not only directly reduce the probability of transmission between susceptible and infected individuals, but they also provide indirect protection for at-risk individuals through herd immunity [8]. Herd immunity is established when a certain proportion of the population is immune to the disease in question, either through vaccination or disease exposure, and transmission is sufficiently reduced so that susceptible individuals avoid infection [9]. The threshold for the proportion of a population that needs to be immune to achieve herd immunity partly depends on the transmissibility of a disease, measured by the basic reproduction number,

2 Department of Medicine, Tufts University School of Medicine, Boston, MA, USA 
$\mathrm{R}_{0} . \mathrm{R}_{0}$ represents the number of secondary infections generated from the very first infection in the completely susceptible population, which varies from disease to disease [8]. A larger value of $\mathrm{R}_{0}$ requires the higher proportion of immune individuals to achieve herd immunity.

Public health vaccination programs, in particular those in low- and middle-income countries (LMICs), often use economic evaluations to inform funding priorities and maximize health gains. LMICs face multiple competing health challenges, such as other communicable diseases and an increasing burden of chronic diseases [10,11]. The significance of economic evaluations of vaccine programs may be greater in LMICs because these countries must often choose between vaccination strategies and other health programs given the extremely limited resources available.

The World Health Organization (WHO) recommends that vaccine economic evaluations include herd immunity in the analyses, particularly in situations when ignoring such effects would result in a substantial difference in the estimated cost-effectiveness of the program [12]. Costeffectiveness analyses (CEAs) typically incorporate herd immunity using two general modeling approaches [13]. The first approach is to simulate the impact of vaccination with a "dynamic model," which can capture both direct and indirect effects (i.e., herd immunity) of vaccines by simulating pathogen transmission between susceptible individuals. The other approach is to use a "static model," which does not reflect disease transmission because the model assumes a constant risk of infection. In this case, herd immunity is estimated by adding a fixed percentage improvement in the vaccine effectiveness model output, altering model inputs, or applying a multiplier to vaccine efficacy.

Although herd immunity is a key benefit of vaccination programs, the effects are not routinely included in costeffectiveness studies. In a systematic review of 625 vaccine economic evaluations worldwide, only $28 \%$ included herd immunity [13]. To date, it remains unclear how much cost-effectiveness results may change when herd immunity is included, and how often study conclusions could change given various cost-effectiveness thresholds that are employed in LMICs. In most cases, we expect that including herd immunity effects would lead to more favorable estimates of cost-effectiveness. However, there are rare cases where including herd immunity in a CEA could make an intervention less cost-effective. For example, pneumococcal vaccines (e.g., PCV7, PCV10, PCV13) target at most 13 of the more than 92 pneumococcal serotypes. Achieving herd immunity among those 13 serotypes may lead to serotype replacement, which may in turn increase the non-vaccine serotype prevalence, thus reducing the benefits of vaccination. Beyond these broad illustrations, however, additional research is needed to quantify the impact of herd immunity effects on CEA results, and determine how often there may be implications for policies that are adopted based on these findings.

As the COVID-19 pandemic continues to spread globally, the numbers of confirmed cases are rising rapidly in many LMICs [14]. LMICs are particularly at risk of COVID-19 given their low-resourced health systems and multiple competing health challenges. It will be important to evaluate the economic value of COVID-19 vaccines so that LMIC decision makers will have the right information to make funding prioritization decisions. In fact, pneumococcal vaccine findings may be an important analog for COVID-19 given pneumonia presents as the most severe manifestation of disease.

The objectives of this study were to determine: (1) the frequency with which herd immunity effects have been incorporated into economic evaluations of vaccines in LMICs; (2) the characteristics of CEA studies with versus without herd immunity effects; (3) how the inclusion of herd immunity effects changes CEA results under various cost-effectiveness benchmarks by presenting ICERs with and without these effects; and (4) how the inclusion of herd immunity effects impacts the cost-effectiveness of pneumococcal vaccines, to illustrate potential implications for COVID-19 vaccines.

\section{Methods}

\subsection{Data Source and Study Selection}

We searched for economic evaluations of vaccines published between 2000 and 2018 in two distinct data sources: the Tufts Medical Center Cost-effectiveness Analysis Registry (CEAR) and the Global Health CEAR. Both registries are maintained by the Center for the Evaluation of Value and Risk in Health at Tufts Medical Center in Boston, Massachusetts. The CEAR (www.cearegistry.org) contains detailed information on published, English-language cost per qualityadjusted life-year (QALY) studies from 1976 to 2020 and the Global Health CEAR (www.ghcearegistry.org ) summarizes published, English-language cost per disability-adjusted life-year (DALY) studies from 1996 to 2020. The search strategies, inclusion criteria, and review methods for both registries have been published elsewhere [15-18]. Briefly, the procedure for CEAR begins with an electronic literature database search using the keywords that include "QALYs," "quality-adjusted life-year," and "cost-utility." Following this, abstracts are screened for articles that contain cost per QALY results. Selected studies undergo full paper review by two independent reviewers who extract information for inclusion in the CEAR on study methods, incremental costeffectiveness ratios (ICERs) and utility weights. The process for the Global Health CEAR is nearly identical, except for the use of "DALYs" and "disability-adjusted life year" in the search strategy. 
We searched both databases and applied the following inclusion criteria: (1) CEA studies published between 2000 and 2018; (2) analyses that studied vaccination as an intervention (e.g., vaccination vs. no vaccination, or comparing alternative vaccines); (3) a target population in one or more LMICs based on the World Bank's country classification [19] (Fig. 1). We chose the years 2000-2018 to focus on more current studies, and because 2018 was the most current year of studies contained in both registries at the time of this analysis.

\subsection{Analysis}

We investigated the proportion of vaccine CEAs that incorporated herd immunity effects into their analyses and identified the methods used for quantifying such effects. We compared characteristics (i.e., publication year, funding source, disease of focus, study quality) of vaccine CEAs with versus without herd immunity effects. The funding source was important to investigate because different funders may have different incentives to include or exclude herd immunity effects in CEAs. For example, vaccine manufacturers may prefer to include broader benefits of vaccines to make value arguments, whereas governments may prefer more conservative estimates to prioritize health services using a consistent perspective for resource allocation purposes. We assessed study quality using a subjective rating score assigned by Registry reviewers, ranging from 1 (lowest quality) to 7 (highest quality). Scores are aligned with seven principles of economic evaluation methods and reporting, and studies are given 1 point for each of the following: (1) Methods and results were communicated clearly and transparently to enable easy interpretation; (2) Time horizon was of sufficient length to capture all differences in costs and health benefits and discount rate was specific to the local setting; (3) Detailed disaggregated cost and QALY information was provided and reported ICER was correct; (4) Comprehensive characterization of uncertainty (sensitivity analysis) was undertaken; (5) Explicit reporting of utility weights (includes utility weight value and estimation method); (6) Subgroup analysis performed where relevant; and (7) Nonhealth effects and/or spillover effects were quantified [20].

In addition, we examined how incorporating herd immunity effects impacted the results of a CEA. When possible within a study, we compared an ICER that included herd immunity effects with a corresponding ICER that did not include such effects. We used herd immunity effects reported in the original papers. Some of these studies did not empirically quantify herd immunity effects but extrapolated from previously published data or assumptions. When those comparisons were not readily available in the paper, but disaggregated costs and effects were, we recalculated the reported ICER to create two ICERs with and without herd immunity effects. We recorded whether including herd immunity effects would increase or decrease the ICER and by how much, and whether the change in ICER was significant enough to cause the results to cross commonly cited CEA benchmarks for LMICs: cost-saving (less costly and more effective); $1 \times$ gross domestic product (GDP) per capita, $3 \times$ GDP per capital, dominated (more costly and less effective) $[21,22]$. We further explored the impact of herd immunity in a subset of studies focusing on vaccines against pneumococcal disease. We conducted this case study because these vaccines protect against a form of pneumonia, which is a primary consequence of COVID-19. This case study may help provide context for the consideration of herd immunity when determining the economic value of COVID-19 vaccines. All ICERs and GDP per capita estimates were converted to 2020

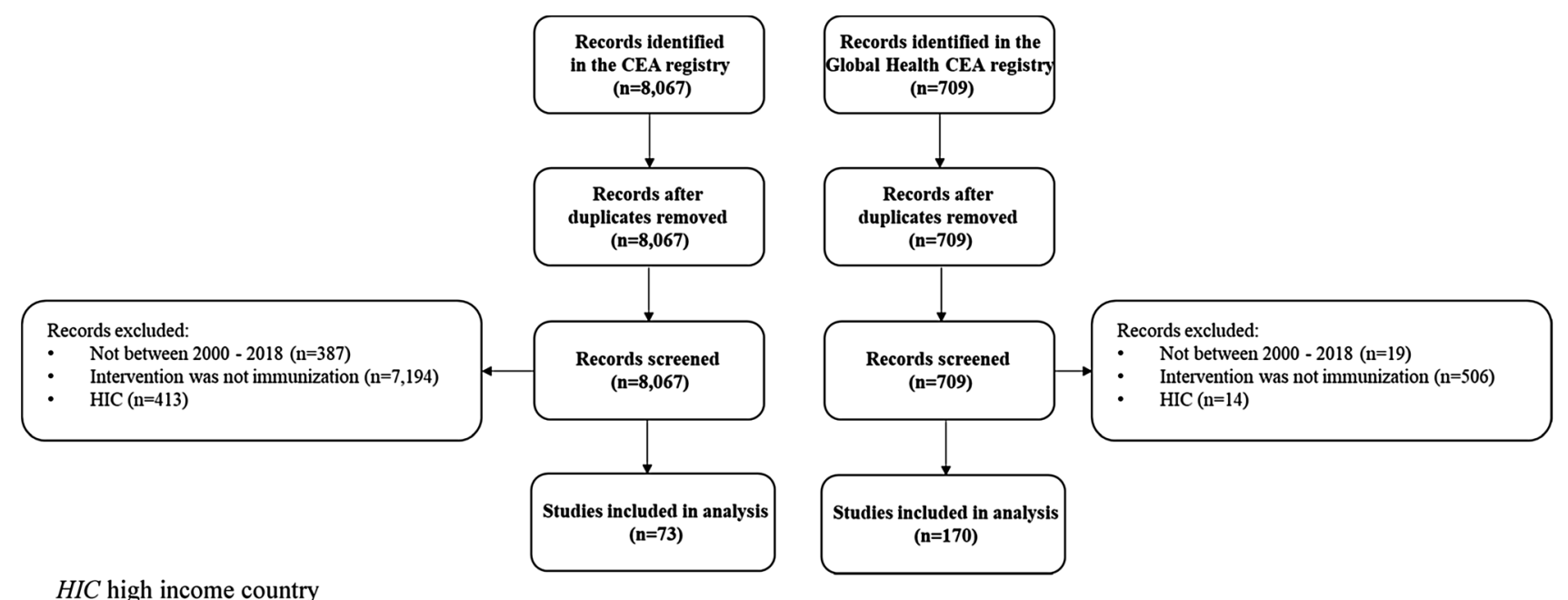

Fig. 1 Literature review flow diagram. HIC high income country 
US dollars using relevant currency exchange rates and the medical care component of the US consumer price index (https://www.bls.gov/cpi/).

\section{Results}

\subsection{Characteristics of Vaccine Cost-Effectiveness Analyses}

Among the 8,067 CEA studies in the CEAR and 709 CEAs in the Global Health CEAR, we excluded 7,994 and 539 studies, respectively, that did not meet our inclusion criteria. Our final analysis included 243 vaccine CEAs, with 73 cost-per-QALY studies and 170 cost-per-DALY studies (Fig. 1). The number of vaccine CEAs increased over time. Almost half of the studies ( $n=118 ; 48 \%$ ) reported government or foundation funding. The most commonly studied disease areas were rotavirus (22\%), followed by HPV (17\%) and pneumococcal disease (17\%). Approximately $77 \%$ of the included studies were given a quality score of 5 or greater (range 1-7).

\subsection{Estimating Herd Immunity Effects in Vaccine Cost-effectiveness Analyses}

We found that 44 vaccine CEAs (18\% of 243) included herd immunity effects, including 16 (22\% of 73) cost-perQALY studies and 28 (16\% of 170) cost-per-DALY studies. Among vaccine CEAs that included herd immunity effects, $11 \%$ were published in 2000-2010, 32\% published in 2011-2014, and 57\% published in 2015-2018. Among vaccine CEAs that did not include herd immunity effects, the number of studies published in each period was more evenly distributed. Somewhat surprisingly, studies funded by governments or foundations included herd immunity effects more frequently (55\%) than those funded by industry $(20 \%)$ or other sources (7\%). The majority of studies that incorporated herd immunity effects evaluated pneumococcal vaccines (43\%), while those that did not include herd immunity effects mainly studied rotavirus (Table 1).

Of the 44 studies that included herd immunity effects, 11 (25\%) used dynamic transmission models, while the remainder used static models. For studies that used dynamic models, we were unable to disentangle the impact of herd immunity from other vaccine benefits because herd immunity effects were not reported separately. In the 33 static models, herd immunity was estimated by adding a fixed percentage improvement in the vaccine effectiveness model output in 18 studies (54\%) [23-30], altering model inputs (i.e., assuming a percentage decrease in disease incidence) in ten studies (30\%) [31-35], or applying a multiplier to vaccine efficacy in three studies (9\%) [36]. Two static model studies (6\%) did not clearly state how herd immunity effects were estimated [37, 38] (Table 2). Among the 33 static models, 16 reported detailed data or allowed us to recalculate and compare each ICER alternatively with and without the inclusion of herd immunity ( $n=48$ ratio pairs). Within this subsample, there were eight studies that focused on vaccines for pneumococcal disease.

\subsection{Impact of Herd Immunity Effects on the Reported Incremental Cost-Effectiveness Ratio}

The inclusion of herd immunity effects made all 48 ICERs more favorable, including five cases with cost-saving results that remained so after adjustment. In 20 cases (42\%), adding herd immunity effects decreased the ICER enough to cross at least one or more commonly cited cost-effectiveness benchmarks for LMICs; in six cases, interventions that were originally cost-increasing became cost-saving (Table 3). After excluding results that became cost-saving with herdimmunity $(n=11)$, the average ICER without herd immunity was \$55,552/QALY (range: \$600/QALY-\$613,691/ QALY); the inclusion of herd immunity on average reduced the ICER to $\$ 41,074$ (or by $28 \%$ ). Among DALY-based studies, the mean ICER without herd immunity was $\$ 11,211$ / DALY (range \$52/DALY-\$69,411/DALY); including herd immunity reduced the average ICER to $\$ 6470$ (or by $61 \%$ ) (Table 4).

To illustrate how the inclusion of herd immunity effects in a CEA may affect the value of COVID-19 vaccines, we calculated 24 ICERs with and without herd immunity from eight studies that examined the cost-effectiveness of the pneumococcal vaccine compared to no vaccine in a pediatric population. In three cases, the pneumococcal vaccine was estimated to be cost-saving even without herd immunity effects. In eight cases (33\%), adding herd immunity effects decreased the ICER enough to cross at least one commonly cited cost-effectiveness benchmark for LMICs; in most of these, the result became cost-saving (Table 3). After excluding interventions that were cost-saving with the inclusion of herd immunity $(n=8)$, the mean QALYbased ICER without herd immunity was \$61,789/QALY (range \$600/QALY-\$613,691/QALY), and the inclusion of herd immunity on average reduced the ICER to $\$ 48,056$ (or by $31 \%$ ). The mean DALY-based ICER without herd immunity was \$2,681/DALY (range \$87/DALY-\$6905DALY) and was reduced to $\$ 1728$ (or by $49 \%$ ) on average with herd immunity (Table 4 ). The ICER differences with vs. without herd immunity stratified by World Bank's country income classification (i.e., low-, lower middle-, upper middle-income countries) can be found in the Electronic Supplementary Material (ESM) Table 1. The results 
Table 1 Characteristics of vaccine cost-effectiveness analyses ( $n=243$, all studies)

\begin{tabular}{|c|c|c|c|}
\hline & Total & $\begin{array}{l}\text { Herd immunity effects } \\
\text { included, } n(\%)\end{array}$ & $\begin{array}{l}\text { No herd immu- } \\
\text { nity effects, } n \\
(\%)\end{array}$ \\
\hline$N(\%)$ & $243(100)$ & $44(18)$ & $199(82)$ \\
\hline \multicolumn{4}{|l|}{ Publication year } \\
\hline 2000-2010 & $65(27)$ & $5(11)$ & $60(30)$ \\
\hline 2011-2014 & $70(29)$ & $14(32)$ & $56(28)$ \\
\hline $2015-2018$ & $108(44)$ & $25(57)$ & $83(42)$ \\
\hline \multicolumn{4}{|l|}{ Funding source } \\
\hline Government or foundation & 118 (48) & $24(55)$ & $94(47)$ \\
\hline Industry & $38(16)$ & $9(20)$ & $29(15)$ \\
\hline None/not determined & $64(26)$ & $8(18)$ & $56(28)$ \\
\hline Other & $23(10)$ & $3(7)$ & $20(10)$ \\
\hline \multicolumn{4}{|l|}{ Disease } \\
\hline Rotavirus & $53(22)$ & $6(14)$ & $47(24)$ \\
\hline HPV & $42(17)$ & $4(9)$ & $38(19)$ \\
\hline Pneumococcal disease & $41(17)$ & $19(43)$ & $22(11)$ \\
\hline Hepatitis & $21(8)$ & $0(0)$ & $21(11)$ \\
\hline Influenza & $19(8)$ & $3(7)$ & $16(8)$ \\
\hline Other $^{\mathrm{a}}$ & $67(28)$ & $12(27)$ & $55(6)$ \\
\hline \multicolumn{4}{|l|}{ Quality score (range 1-7) } \\
\hline$\leq 4$ & $62(23)$ & $5(11)$ & $57(29)$ \\
\hline$>4$ & 181 (77) & $39(89)$ & $142(71)$ \\
\hline
\end{tabular}

${ }^{a}$ Other category includes disease areas that have six or fewer studies in total, including Encephalitis (6), Rabies (6), Multiple disease areas (6), Dengue (5), Malaria (5), Measles (5), Cholera (4), Herpes zoster virus (4), Typhoid (4), Meningitis (3), Polio (3), Tetanus (3), Enterovirus 71 (2), Zika virus (2), brucellosis (1), Chagas disease (1), Echinococcosis (1), Hookworm (1), Norovirus (1), Q fever (1), Respiratory syncytial virus (1), Tuberculosis (1), Valley fever (1)

\begin{tabular}{llc}
\hline & $\begin{array}{l}\text { Included herd immunity } \\
\text { effects, } n(\%)\end{array}$ & $\begin{array}{l}\text { Reported ICER with and with- } \\
\text { out herd immunity effects, } n(\%)\end{array}$ \\
\hline Total number of studies & $44(100)$ & $16(100)$ \\
Dynamic transmission model & $11(25)$ & $0(0)$ \\
Static model & $33(75)$ & $16(100)$ \\
$\quad$ Decrease in incidence of the disease & $10(30)$ & $4(25)$ \\
Increased vaccine efficacy & $3(9)$ & $3(19)$ \\
Increase of direct vaccine impact on & $18(54)$ & $9(56)$ \\
$\quad$ health outcome & $2(6)$ & $0(0)$ \\
$\quad$ Not stated &
\end{tabular}

Table 2 Methods for estimating herd immunity effects
LMICs [12, 39], our review found that less than one-fifth of vaccine studies in our sample did so. This percentage is lower than the percentage reported in a previous systematic review [13]. The discrepancy may be due to the exclusion of studies focused on high-income countries from our sample that were included in the previous review. We found that studies that included herd immunity effects consistently had more favorable cost-effectiveness results, with ICER reductions ranging from 28 to $61 \%$. 
Table 3 Change in cost-effectiveness analysis value determinations when herd immunity effects are incorporated

\begin{tabular}{|c|c|c|}
\hline & All studies, $n(\%)$ & $\begin{array}{l}\text { Pneumococcal } \\
\text { vaccine studies, } \\
n(\%)\end{array}$ \\
\hline Number of studies reported ICERs with and without HI effects ${ }^{\mathrm{a}}$ & 16 (of 44 studies) & 8 (of 16 studies) \\
\hline Number of ICERs recalculated with/without herd immunity effects ${ }^{\mathrm{b}}$ & 48 (of 140 ICERs) & 24 (of 48 ICERs) \\
\hline \$/QALY & $21(44)$ & $18(75)$ \\
\hline \$/DALY & $27(56)$ & $6(25)$ \\
\hline \multicolumn{3}{|l|}{ Where herd immunity effects included } \\
\hline Base case & $25(52)$ & $7(29)$ \\
\hline Sensitivity analysis & $24(50)$ & $18(75)$ \\
\hline Both & $1(2)$ & $1(4)$ \\
\hline \multicolumn{3}{|l|}{ Change in ICER after adding herd immunity effects } \\
\hline Increase & $0(0)$ & $0(0)$ \\
\hline Decrease & $48(100)$ & $24(100)$ \\
\hline No change & $0(0)$ & $0(0)$ \\
\hline \multicolumn{3}{|l|}{ Including HI reduced the ICER enough to cross the following thresholds ${ }^{c}$} \\
\hline \multicolumn{3}{|l|}{ \$/DALY } \\
\hline Cost-saving threshold & $2(7)$ & $1(17)$ \\
\hline $1 \times$ GDP threshold & $10(37)$ & $0(0)$ \\
\hline $3 \times$ GDP threshold & $8(30)$ & $2(33)$ \\
\hline Did not cross any threshold & $11(41)$ & $3(50)$ \\
\hline \multicolumn{3}{|l|}{ \$/QALY } \\
\hline Cost-saving threshold & $4(19)$ & $4(22)$ \\
\hline $1 \times$ GDP threshold & $6(25)$ & $6(33)$ \\
\hline $3 \times$ GDP threshold & $0(0)$ & $0(0)$ \\
\hline Did not cross any threshold & $17(81)$ & $13(72)$ \\
\hline
\end{tabular}

$I C E R$ incremental cost-effectiveness ratio, $Q A L Y$ quality-adjusted life-year, $D A L Y$ disability-adjusted life-year, $H I$ herd immunity, $G D P$ gross domestic production

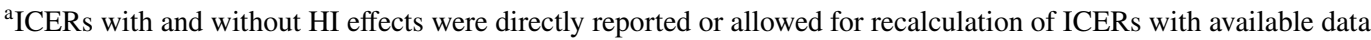

${ }^{b}$ Recalculated ICERs refer to a pair of ICERs from the same study, one that includes and the other without herd immunity effects

${ }^{c}$ Not mutually exclusive: including herd immunity may cause the ICER results to cross more than one threshold (e.g., ICER results changed from dominated to below $1 \times$ GDP threshold, the results were counted in both $1 \times$ GDP threshold and $3 \times$ GDP threshold categories)

Table 4 The mean ICERs without herd immunity and the average percentage reduction in ICERs with the inclusion of herd immunity

\begin{tabular}{cllll}
\hline & ICER & Without HI effects & $\begin{array}{l}\text { Actual ICER } \\
\text { reduction with HI }\end{array}$ & $\begin{array}{l}\text { Percentage reduction in } \\
\text { ICER with HI (mean) } \\
(\%)\end{array}$ \\
\hline $\begin{array}{c}\text { All studies } \\
\text { (mean) }\end{array}$ & \$/QALY $(n=14)$ & $\$ 55,552$ & $\$ 41,074$ & 28 \\
$\begin{array}{c}\text { Pneumo- } \\
\text { coccal }\end{array}$ & \$/QALY $(n=23)$ & $\$ 11,211$ & $\$ 6,470$ & 61 \\
$\begin{array}{l}\text { Disease } \\
\text { (mean) }\end{array}$ & \$/DALY $(n=4)$ & $\$ 61,789$ & $\$ 48,056$ & 31 \\
\hline
\end{tabular}

ICER incremental cost-effectiveness ratio, $H I$ herd immunity, $Q A L Y$ quality-adjusted life-year, $D A L Y$ disability-adjusted life-year

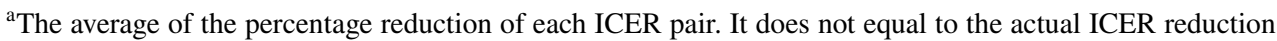
with HI over the ICER without HI effects

${ }^{\mathrm{b}}$ Cost-saving results are not included 
LMICs face severe resource limitations and competing public health priorities. Therefore, studies must provide evidence of the economic and social value of vaccinations in LMICs to help decision-makers appropriately prioritize these interventions. Our data show that in $42 \%$ of cases, the reductions in ICERs with the inclusion of herd immunity effects were large enough to cross at least one or more common cost-effectiveness benchmarks, which could lead to important changes in policy decisions on whether to pay for an intervention based on its economic value. The same trends hold true for pneumococcal vaccine CEAs, which may be an important analog for COVID-19 vaccines. Measuring the full benefits that vaccines confer on society through herd immunity provides important information to policy makers and funders as they prioritize the allocation of scarce resources in LMICs.

Among those studies that included herd immunity in their models, one-quarter used dynamic models to simulate disease transmission in the population, while the remainder used static models. Studies using dynamic transmission models may more accurately capture the impact of herd immunity because they simulate pathogen transmission among the susceptible population; however, the reports on the dynamic models in our sample did not provide sufficient information to understand herd immunity effects. In contrast, static models are unable to capture disease transmission among individuals, and there is no standardized or generally accepted method to quantify herd immunity effects using these models. In our sample, the studies with static models approximated herd immunity effects by altering model inputs, such as assuming a percentage decrease in disease incidence, applying a multiplier to vaccine efficacy, or by adding a fixed percentage improvement in vaccine effectiveness outputs. However, the rationale for the specific method used in these static models was generally not explained clearly. Future vaccine CEAs should strive to incorporate and estimate herd immunity effects accurately and transparently, regardless of the method employed.

Currently, there is a lack of international policy agreement on whether herd immunity effects should be included in economic evaluations of vaccinations. The Advisory Committee on Immunization Practices (ACIP) guidelines in the USA consider herd immunity a function of the capabilities and acceptance of the vaccination program rather than the effectiveness of the vaccine itself. Before a new vaccine enters the US market, ACIP requires the base-case CEA results to exclude herd immunity for a more conservative estimation of vaccine benefits and include herd immunity effects in a sensitivity analysis [40]. On the other hand, the WHO and European guidelines suggest including herd immunity in situations where its exclusion would make a substantial difference in the estimated cost-effectiveness of the program $[12,41]$. It is important to note the differences among these guidelines, as our study showed that CEA results may in some cases change substantially when herd immunity is considered. Since CEA is a key element in health technology assessment, it is important to show the results both with and without herd immunity to accurately estimate the value of a vaccination under all scenarios, as well as to gauge whether its inclusion makes a material difference.

We used pneumococcal vaccination as a case study to examine potential implications for COVID-19 because pneumonia is a primary symptom of COVID-19 infection. However, it is important to note that there are differences between pneumococcal diseases and COVID-19 that limit their comparability. Even though pneumonia is a primary consequence of COVID-19, current estimates of the basic reproductive number $\left(\mathrm{R}_{0}\right)$ for COVID-19 is much higher than that for pneumococcal disease. The $\mathrm{R}_{0}$ for pneumococcal disease is approximately two [42], meaning in a fully susceptible population, each pneumonia case on average infects two additional people. In order to achieve herd immunity, $50 \%$ of the population would need to be vaccinated to prevent population spread. Precise estimates of the $\mathrm{R}_{0}$ for COVID-19 are still evolving. The initial $R_{0}$, based on early estimation from the COVID-19 epicenter in Wuhan, China, was estimated to be 2.2-2.7 [43]. However, a recent study published by the Centers for Disease Control and Prevention in the USA showed that the $\mathrm{R}_{0}$ could be as high as 5.7 in some settings [44]. In this case, over $80 \%$ of the population would need to be vaccinated to achieve herd immunity. Therefore, herd immunity effects will be easier to achieve and will have more impact on the value of vaccines for pneumococcal diseases than they will for COVID-19 if these new $\mathrm{R}_{0}$ estimates stand.

Despite the need for a significant percentage of the population to be vaccinated against COVID-19 to achieve herd immunity, global vaccine coverage rates remain low [45]. Vaccine hesitancy, the phenomenon in which individuals avoid vaccines because they consider them unsafe and/or unnecessary, is considered a growing threat to the success of vaccination programs [46]. Recent data show that the percentage of people in the USA who are committed to receiving the COVID-19 vaccine remains low (50\% as of June 2020 [47, 48], increasing to $69 \%$ as of March 2021 [49]). While vaccine hesitancy is of less concern for LMICs [50, 51], the more pressing issue in these countries is limited access to vaccines. Future studies should explore how vaccine access affects the potential to achieve herd immunity for COVID-19 in LMICs.

Our review is limited by the methods and models developed by the authors in the published CEAs included in our study sample. Although dynamic models are considered the gold standard for modeling infectious disease [12, 41], we 
were unable to include studies with dynamic models to analyze how the inclusion of herd immunity effects changes ICERs, because those studies did not report sufficient information to replicate these estimates. In addition, due to the small sample size, we were unable to tease out how herd immunity effects vary by different types of vaccine CEAs (i.e., a hypothetical vaccine, a replacement vaccine, alternative vaccine strategies, or a different target population). Our study sample was also limited to published and Englishlanguage manuscripts. Technical reports from influential immunization organizations such as the National Immunization Technical Advisory Groups (NITAG), ACIP, and other health technology assessment organizations were not included. Finally, we included studies of all quality in the initial study sample, so it is possible that lower quality studies have findings that are not as verifiable. We conducted a sensitivity analysis that excluded low quality studies (i.e., study score $\leq 4)$. Most results remained the same, except that there were fewer studies funded by industry (10\% vs. $16 \%$ in the full sample). These results are shown in ESM Table 2. (All studies reporting herd immunity effects were rated as having a quality score greater than 4 . Therefore, the effect of including herd immunity was the same as Table 4 in the manuscript.)

\section{Conclusion}

Although herd immunity is an important benefit of vaccination programs, the majority of vaccine CEAs in LMICs do not incorporate herd immunity in their analysis. As a result, these CEAs may underestimate the potential value of vaccines when herd immunity is achieved. Given the evidence in CEAs of the pneumococcal vaccine, an important analog for COVID-19, that showed that accounting for herd immunity effects led to reductions in incremental cost-effectiveness ratios of 31-49\%, COVID-19 vaccination value assessment should aim to produce results both with and without herd immunity effects for decision-making in LMICs.

Supplementary Information The online version contains supplementary material available at https://doi.org/10.1007/s40258-021-00711-y.

\section{Declarations}

Funding This study was performed with financial support from the Bill and Melinda Gates Foundation (OPP1171680). The views expressed herein are those of the authors and do not necessarily reflect the official policy or position of the Bill and Melinda Gates Foundation.

Conflict of interest The authors report grants from the Gates Foundation during the conduct of this study. The authors are affiliated with the CEA Registry, which receives unrestricted sponsorships from a number of government, private foundation, and pharmaceutical industry sponsors (list is available at https://cevr.tuftsmedicalcenter.org/spons orship). DAO and SM report grants from GlaxoSmithKline, outside of the submitted work. DAO reports personal fees from the Center for Global Development, EMD Serono, Amgen, Analysis Group, Aspen Institute/University of Southern California, GalbraithWight, personal fees from Cytokinetics, Executive Insight, Sunovion, University of Colorado, AstraZeneca, Neurocrine, Alkermes, outside of the submitted work. TAL reports personal fees from Merck, outside of the submitted work. PJL reports no additional competing interests.

Ethics approval Not applicable.

Consent to participate Not applicable.

Consent for publication Not applicable.

Availability of data and materials The datasets generated and/or analyzed during the current study are available in the CEA Registry and the Global Health CEA Registry, which is held by the Center for the Evaluation of Value and Risk in Health at Tufts Medical Center. Limited data from the CEA Registry are publicly available at www.ceare gistry.org. Data from the Global Health CEA Registry are publicly available at www.ghcearegistry.org.

Code availability Not applicable.

Author contributions All authors meet the four ICMJE criteria for authorship. SM conceptualization, methodology, software, validation, formal analysis, data curation, writing-original draft preparation, visualization. TAL conceptualization, writing - review and editing. DAO writing - review and editing, funding acquisition. PL conceptualization, writing - review and editing, supervision.

Open Access This article is licensed under a Creative Commons Attribution-NonCommercial 4.0 International License, which permits any non-commercial use, sharing, adaptation, distribution and reproduction in any medium or format, as long as you give appropriate credit to the original author(s) and the source, provide a link to the Creative Commons licence, and indicate if changes were made. The images or other third party material in this article are included in the article's Creative Commons licence, unless indicated otherwise in a credit line to the material. If material is not included in the article's Creative Commons licence and your intended use is not permitted by statutory regulation or exceeds the permitted use, you will need to obtain permission directly from the copyright holder. To view a copy of this licence, visit http://creativecommons.org/licenses/by-nc/4.0/.

\section{References}

1. Clark AD, Griffiths UK, Abbas SS, Rao KD, Privor-Dumm L, Hajjeh R, Johnson H, Sanderson C, Santosham M. Impact and cost-effectiveness of Haemophilus influenzae type b conjugate vaccination in India. J Pediatr. 2013;163(1):S60-72.

2. Griffiths UK, Clark A, Hajjeh R. Cost-effectiveness of Haemophilus influenzae type $\mathrm{b}$ conjugate vaccine in low-and middle-income countries: regional analysis and assessment of major determinants. J Pediatr. 2013;163(1):S50-9.

3. Le P, Griffiths UK, Anh DD, Franzini L, Chan W, Swint JM. Cost-effectiveness of Haemophilus influenzae type $\mathrm{b}$ vaccine in Vietnam. Vaccine. 2015;33(36):4639-46.

4. Gargano LM, Hajjeh R, Cookson ST. Pneumonia prevention during a humanitarian emergency: cost-effectiveness of Haemophilus influenzae type b conjugate vaccine and 
pneumococcal conjugate vaccine in Somalia. Prehosp Disaster Med. 2015;30(4):402-11.

5. Rheingans R, Amaya M, Anderson J, Chakraborty P, Atem J. Systematic review of the economic value of diarrheal vaccines. Hum Vaccin Immunother. 2014;10(6):1582-94.

6. Paternina-Caicedo A, De la Hoz-Restrepo F, Alvis-Guzmán N. Epidemiological and economic impact of monovalent and pentavalent rotavirus vaccines in low and middle income countries: a cost-effectiveness modeling analysis. Pediatr Infect Dis J. 2015;34(7):e176-84.

7. Sibak M, Moussa I, El-Tantawy N, Badr S, Chaudhri I, Allam E, Baxter L, Freikha SA, Hoestlandt C, Lara C. Cost-effectiveness analysis of the introduction of the pneumococcal conjugate vaccine (PCV-13) in the Egyptian national immunization program, 2013. Vaccine. 2015;33:A182-91.

8. Metcalf CJE, Ferrari M, Graham AL, Grenfell BT. Understanding herd immunity. Trends Immunol. 2015;36(12):753-5.

9. Fine P, Eames K, Heymann DL. "Herd immunity": a rough guide. Clin Infect Dis. 2011;52(7):911-6.

10. Abegunde DO, Mathers CD, Adam T, Ortegon M, Strong K. The burden and costs of chronic diseases in low-income and middle-income countries. Lancet. 2007;370(9603):1929-38.

11. World Health Organization. Chronic disease in low and middle income countries. https://www.who.int/chp/chronic_disease_ report/media/Factsheet3.pdf. Accessed 20 Oct 2021.

12. World Health Organization. WHO guide for standardization of economic evaluations of immunization programmes. Geneva: World Health Organization; 2019.

13. Nymark LS, Sharma T, Miller A, Enemark U, Griffiths UK. Inclusion of the value of herd immunity in economic evaluations of vaccines. A systematic review of methods used. Vaccine. 2017;35(49):6828-41.

14. World Health Organization. Coronavirus disease 2019 (COVID19): situation report, 177. 2020.

15. Neumann PJ, Greenberg D, Olchanski NV, Stone PW, Rosen AB. Growth and quality of the cost-utility literature, 19762001. Value Health. 2005;8(1):3-9.

16. Neumann PJ, Thorat T, Shi J, Saret CJ, Cohen JT. The changing face of the cost-utility literature, 1990-2012. Value Health. 2015;18(2):271-7.

17. Neumann PJ, Thorat T, Zhong Y, Anderson J, Farquhar M, Salem M, Sandberg E, Saret CJ, Wilkinson C, Cohen JT. A systematic review of cost-effectiveness studies reporting Costper-DALY averted. PLoS ONE. 2016;11(12):e0168512.

18. Neumann PJ, Anderson JE, Panzer AD, Pope EF, D'Cruz BN, Kim DD, Cohen JT. Comparing the cost-per-QALYs gained and cost-per-DALYs averted literatures. Value Health. 2018;21:S118.

19. World Bank Country and Lending Groups. https://datahelpde sk.worldbank.org/knowledgebase/articles/906519-world-bankcountry-and-lending-groups\#: :text=For $\% 20$ the $\% 20$ current $\%$ $202021 \% 20$ fiscal,those $\% 20$ with\%20a\%20GNI\%20per.

20. The Cost-Effectiveness Analysis Registry [Internet]. (Boston), Institute for Clinical Research and Health Policy Studies, Tufts Medical Center. www.cearegistry.org.

21. Ivinson AJ. Macroeconomics and health: investing in health for economic development. Nature Med. 2002;8(6):551-2.

22. Leech AA, Kim DD, Cohen JT, Neumann PJ. Use and misuse of cost-effectiveness analysis thresholds in low-and middle-income countries: trends in cost-per-DALY studies. Value in Health. 2018;21(7):759-61

23. Kim S-Y, Lee G, Goldie SJ. Economic evaluation of pneumococcal conjugate vaccination in the Gambia. BMC Infect Dis. 2010;10(1):260.

24. Griffiths UK, Clark A, Shimanovich V, Glinskaya I, Tursunova D, Kim L, Mosina L, Hajjeh R, Edmond K. Comparative economic evaluation of Haemophilus influenzae type b vaccination in Belarus and Uzbekistan. PLoS ONE. 2011;6(6):e21472.

25. Schaetti C, Weiss MG, Ali SM, Chaignat C-L, Khatib AM, Reyburn R, Tebbens RJD, Hutubessy R. Costs of illness due to cholera, costs of immunization and cost-effectiveness of an oral cholera mass vaccination campaign in Zanzibar. PLoS Negl Trop Dis. 2012;6(10):e1844.

26. Caldwell R, Roberts CS, An Z, Chen C-I, Wang B. The health and economic impact of vaccination with 7-valent pneumococcal vaccine (PCV7) during an annual influenza epidemic and influenza pandemic in China. BMC Infect Dis. 2015;15(1):284.

27. Bakir M, Standaert B, Turel O, Bilge ZE, Postma M. Estimating and comparing the clinical and economic impact of paediatric rotavirus vaccination in Turkey using a simple versus an advanced model. Vaccine. 2013;31(6):979-86.

28. Martí SG, Colantonio L, Bardach A, Galante J, Lopez A, Caporale J, Knerer G, Gomez JA, Augustovski F, Pichon-Riviere A. A cost-effectiveness analysis of a 10-valent pneumococcal conjugate vaccine in children in six Latin American countries. Cost Effect Resour Alloc. 2013;11(1):21.

29. Che D, Zhou H, He J, Wu B. Modeling the impact of the 7-valent pneumococcal conjugate vaccine in Chinese infants: an economic analysis of a compulsory vaccination. BMC Health Serv Res. 2014;14(1):56.

30. Zhou H, He J, Wu B, Che D. Cost-effectiveness analysis of routine 13-valent pneumococcal conjugate vaccinations in Chinese infants. Hum Vaccin Immunother. 2018;14(6):1444-52.

31. Haasis MA, Ceria JA, Kulpeng W, Teerawattananon Y, Alejandria $M$. Do pneumococcal conjugate vaccines represent good value for money in a lower-middle income country? A cost-utility analysis in the Philippines. PLoS ONE. 2015;10(7):e0131156.

32. Wu DB-C, Roberts C, Lee VWY, Hong L-W, Tan KK, Mak V, Lee KKC. Cost-effectiveness analysis of infant universal routine pneumococcal vaccination in Malaysia and Hong Kong. Hum Vaccines Immunother. 2016;12(2):403-16.

33. Jeuland M, Cook J, Poulos C, Clemens J, Whittington D, Group DCES. Cost-effectiveness of new-generation oral cholera vaccines: a multisite analysis. Value Health. 2009;12(6):899-908.

34. Sundaram N, Chen C, Yoong J, Luvsan M-E, Fox K, Sarankhuu A, La Vincente S, Jit M. Cost-effectiveness of 13-valent pneumococcal conjugate vaccination in Mongolia. Vaccine. 2017;35(7):1055-63.

35. Shoukat A, Vilches T, Moghadas SM. Cost-effectiveness of a potential Zika vaccine candidate: a case study for Colombia. BMC Med. 2018;16(1):100.

36. Kim S-Y, Choi Y, Mason PR, Rusakaniko S, Goldie SJ. Potential impact of reactive vaccination in controlling cholera outbreaks: an exploratory analysis using a Zimbabwean experience. S Afr Med J. 2011;101(9):659-64.

37. Broughton EI. Economic evaluation of Haemophilus influenzae type B vaccination in Indonesia: a cost-effectiveness analysis. J Public Health. 2007;29(4):441-8.

38. Akumu AO, English M, Scott JAG, Griffiths UK. Economic evaluation of delivering Haemophilus influenzae type b vaccine in routine immunization services in Kenya. Bull World Health Organ. 2007;85:511-8.

39. Jit M, Hutubessy R, Png ME, Sundaram N, Audimulam J, Salim S, Yoong J. The broader economic impact of vaccination: reviewing and appraising the strength of evidence. BMC Med. 2015;13(1):209.

40. Guidance for Health Economics Studies Presented to the Advisory Committee on Immunization Practices (ACIP), 2019 Update. https://www.cdc.gov/vaccines/acip/committee/downloads/Econo mics-Guidance-for-ACIP-2019.pdf. 
41. Ultsch B, Damm O, Beutels P, Bilcke J, Brüggenjürgen B, GerberGrote A, Greiner W, Hanquet G, Hutubessy R, Jit M. Methods for health economic evaluation of vaccines and immunization decision frameworks: a consensus framework from a European vaccine economics community. Pharmacoeconomics. 2016;34(3):227-44.

42. Nurhonen M, Cheng AC, Auranen K. Pneumococcal transmission and disease in silico: a microsimulation model of the indirect effects of vaccination. PLoS ONE. 2013;8(2):e56079.

43. Viceconte G, Petrosillo N. COVID-19 R0: Magic number or conundrum? Infect Dis Rep. 2020;12(1):1-2.

44. Sanche S, Lin YT, Xu C, Romero-Severson E, Hengartner N, Ke R (2020) High contagiousness and rapid spread of severe acute respiratory syndrome coronavirus 2. Emerg infect Dis. 26(7):1470.

45. Immunization coverage. https://www-who-int.ezproxy.library. tufts.edu/news-room/fact-sheets/detail/immunization-coverage.

46. Dubé E, Laberge C, Guay M, Bramadat P, Roy R, Bettinger JA. Vaccine hesitancy: an overview. Hum Vaccin Immunother. 2013;9(8):1763-73.
47. Cornwal W. Just 50\% of Americans plan to get a COVID-19 vaccine. Here's how to win over the rest. Science. 2020. https://www. sciencemag.org/news/2020/06/just-50-americans-plan-get-covid19-vaccine-here-s-how-win-over-rest\#. Accessed 26 Dec 2021.

48. Dror AA, Eisenbach N, Taiber S, Morozov NG, Mizrachi M, Zigron A, Srouji S, Sela E. Vaccine hesitancy: the next challenge in the fight against COVID-19. Eur J Epidemiol. 2020;35(8):775-9.

49. Funk C, Tyson A. Growing share of Americans say they plan to get a COVID-19 vaccine-or already have. Pew Research Center Science \& Society. 2021. Accessed 2 Apr 2021.

50. Organization WH. Report of the SAGE Working Group on Vaccine Hesitancy. Geneva: World Health Organization; 2014.

51. Bhopal S, Nielsen M. Vaccine hesitancy in low-and middleincome countries: potential implications for the COVID-19 response. Arch Dis Child. 2020;106(2):113-4. 